\title{
SOME NEW DETERMINISTIC AND RANDOM VARIATIONAL INEQUALITIES AND THEIR APPLICATIONS
}

\author{
XIAN-ZHI YUAN \\ The University of Queensland \\ Department of Mathematics \\ Brisbane 4072, QLD Australia \\ and \\ Dalhousie University \\ Department of Mathematics \\ Halifax, N.S., B3H 3J5 Canada \\ JEAN-MARC ROY \\ Centre Universitaire de Shippagan \\ Department of Mathematics \\ Shippagan, N.B., E0B $2 P 0$ Canada
}

(Received June, 1994; Revised July, 1995)

\begin{abstract}
A non-compact deterministic variational inequality which is used to prove an existence theorem for saddle points in the setting of topological vector spaces and a random variational inequality. The latter result is then applied to obtain the random version of the Fan's best approximation theorem. Several random fixed point theorems are obtained as applications of the random best approximation theorem.

Key words: $\gamma$-Generalized Quasi-Convex, Random Variational Inequality, Best Approximation, Saddle Point, Generalized KKM Mapping, Random Fixed Point.
\end{abstract}

AMS (MOS) subject classifications: 47H10, 49J40, 54C60, 46C05, 41A50.

\section{Introduction}

As an application of the generalized Knaster-Kuratowski-Mazurkiewicz (KKM) principle, we first establish non-compact deterministic variational inequalities. This result is then used to derive an existence theorem for saddle points in the setting of topological vector spaces. By employing a measurable selection theorem due to Himmelberg [5], a random variational inequality is presented which in turn is applied to derive the random version of th best approximation theorem of Fan [4, Theorem 2]. Finally, as applications of our random best approximation theorem, several random fixed point theorems are given. These results improve and unify corresponding results in the literature.

In this paper, all topological spaces are assumed to be Hausdorff, unless otherwise 
specified. Let $X$ be a non-empty set. We denote by $\mathscr{F}(X)$ the family of all non-empty finite subsets of $X$ and by $\mathscr{P}(X)$, the family of all non-empty subsets of $X$. If $X$ is a non-empty subset of topological space $Y$, the notations $\partial_{Y} X$ (in short, $\partial X$ ) and int $_{Y} X$ (in short, int $Y$ ) denote the boundary and the relative interior of $X$ in $Y$, respectively and $X^{c}:=\{x \in Y: x \notin X\}$. If $A$ is a subset of a vector space $E$, the convex hull of $A$ in $E$ is denoted by $\operatorname{co} A$. We denote by $\mathbb{N}$ and $\mathbb{R}$ the set of all positive integers and the real line, respectively.

A measurable space $(\Omega, \Sigma)$ is a pair, where $\Omega$ is a set and $\Sigma$ is a $\sigma$-algebra of subsets of $\Omega$. If $X$ is a topological space, the Borel $\sigma$-algebra $\beta(X)$ is the smallest $\sigma$-algebra containing all open subsets of $X$. If $\left(\Omega_{1}, \Sigma_{1}\right)$ and $\left(\Omega_{2}, \Sigma_{2}\right)$ are two measurable spaces, the space $\left(\Omega_{1} \times \Omega_{2}, \Sigma_{1} \otimes \Sigma_{2}\right)$ denotes the smallest $\sigma$-algebra which contains all the sets of $A \times B$, where $A \in \Sigma_{1}, B \in \Sigma_{2}$. We note that the Borel $\sigma$-algebra $\beta\left(X_{1} \times X_{2}\right)$ contains $\beta\left(X_{1}\right) \otimes \beta\left(X_{2}\right)$ in general. A mapping $f: \Omega_{1} \rightarrow \Omega_{2}$ is said to be $\left(\Sigma_{1}, \Sigma_{2}\right)$-measurable if for any $B \in \Sigma_{2}, f^{-1}(B):=\left\{x \in \Omega_{1}: f(x) \in B\right\} \in \Sigma_{1}$. Let $X$ be a topological space and $F:(\Omega, \Sigma) \rightarrow \mathscr{P}(X)$ a correspondence (mapping). Then $F$ is said to be $(a)$ measurable if $F^{-1}(B):=\{\omega \in \Omega: F(\omega) \cap B \neq \emptyset\} \in \Sigma$ for each closed subset $B$ of $X$ and (b) have a measurable graph if Graph $F:=\{(\omega, y) \in \Omega \times X: y \in F(\omega)\} \in \Sigma \otimes \beta(X)$. A singlevalued mapping $f: \Omega \rightarrow X$ is said to be a measurable selection of the mapping $F$ if $f$ is a measurable mapping such that $f(\omega) \in F(\omega)$ for all $\omega \in \Omega$.

If $\left(X_{1}, \Sigma_{1}\right)$ and $\left(X_{2}, \Sigma_{2}\right)$ are measurable spaces and $Y$ is topological space, a mapping $F: X_{1}$ $\times X_{2} \rightarrow \mathscr{P}(Y)$ is said to be jointly (resp., jointly weakly) measurable if $f^{-1}(B) \in \Sigma_{1} \otimes \Sigma_{1}$ for each closed (resp., open) subset $B$ of $Y$. When $X$ is a topological space, it is understood that $\Sigma$ is the Borel $\sigma$-algebra $\beta(X)$. Let $X$ and $Y$ be two topological spaces, $(\Omega, \Sigma)$ a measurable space, and $F: \Omega \times X \rightarrow \mathscr{P}(Y)$. Then $F$ is said to be $(i)$ a random operator (mapping) if for each fixed $x \in X$, the mapping $F(\cdot, x): \Omega \rightarrow \mathscr{P}(Y)$ is measurable and (ii) random continuous if for each fixed $\omega \in \Omega$, $F(\omega, \cdot): X \rightarrow \mathscr{P}(Y)$ is continuous and for each fixed $x \in X, F(\cdot, x): \Omega \rightarrow \mathscr{P}(Y)$ is measurable. Let $F: \Omega \times X \rightarrow \mathscr{P}(X)$ be a mapping. Then a single-valued mapping $\phi: \Omega \rightarrow X$ is said to be a random fixed point of $F$ if $\phi$ is a measurable mapping and $\phi(\omega) \in F(\omega, \phi(\omega))$ for all $\omega \in \Omega$. We observe that if $F: \Omega \times X \rightarrow \mathscr{P}(X)$ has a random fixed point then for each fixed $\omega \in \Omega, F(\omega, \cdot)$ has a (deterministic) fixed point in $X$, but the converse does not hold true (e.g., see the example of Tan and Yuan [11]).

It is well-known in the study of convex analysis and its applications that the convex condition plays an essential role (e.g., see the book of Lin and Simons [7] and the references therein). Recently, the concept of convexity was generalized in several ways by Horvath [6], Zhou and Chen [15], and Chang and Zhang [3]. In order to establish our general variational inequalities under weaker convexity we first recall some definitions and facts.

Definition 1.1: Let $X$ be convex subset of a vector space $E$. A function $\phi: X \rightarrow \mathbb{R}$ is said to be quasi-convex (resp., quasi-concave) if the set $\{x \in X: \phi(x) \leq \lambda\}$ (resp., $\{x \in X: \phi(x) \geq \lambda\}$ ) is convex for each $\lambda \in \mathbb{R}$.

We also need the following definition which was introduced by Chang and Zhang [3] and it is a generalization of the classical KKM mapping.

Definition 1.2: Let $X$ and $Y$ be non-empty convex subsets of topological vector spaces $E$ and $F$, respectively. Suppose $G: X \rightarrow \mathscr{P}(F)$ is a set-valued mapping. Then $G$ is said to be a generalized $K K M$ mapping if for each non-empty finite set $\left\{x_{1}, \ldots, x_{n}\right\} \subset X$, there exists a finite set $\left\{y_{1}, \ldots, y_{n}\right\} \subset F$ suc' that for each $\left\{y_{i_{1}}, \ldots, y_{i_{k}}\right\} \subset\left\{y_{1}, \ldots, y_{n}\right\}$, where $1 \leq k \leq n$, the following inclusion holds:

$$
\operatorname{co}\left(y_{i_{1}}, \ldots, y_{i_{k}}\right) \subset \bigcup_{j=1}^{k} G\left(x_{i_{j}}\right) .
$$

We would like to note that the generalized KKM mapping contains the classical KKM mapping as a special case. For more details, see Chang and Zhang [3] and Yuan [14]. 
Definition 1.3: (Chang and Zhang [3]). Let $\gamma \in \mathbb{R}$ be a fixed constant, $X$ and $Y$ non-empty convex subsets of topological vector spaces $E$ and $F$, respectively. A real-valued function $\phi: X \times Y \rightarrow \mathbb{R}$ is said to be $\gamma$-generalized quasi-convex (resp., quasi-concave) on $Y$ if for each finite subset non-empty $\left\{y_{1}, \ldots, y_{n}\right\} \subset Y$, there exists a non-empty finite subset $\left\{x_{1}, \ldots, x_{n}\right\} \subset X$ such that for each $x_{0} \in \operatorname{co}\left\{x_{i_{1}}, \ldots, x_{i_{k}}\right\} \subset\left\{x_{1}, \ldots, x_{n}\right\}$, the following inequality holds:

$$
\gamma \leq \max _{1 \leq j \leq k} \phi\left(x_{0}, y_{i_{j}}\right)\left(\text { resp., } \gamma \geq \min _{1 \leq j \leq k} \phi\left(x_{0}, y_{i j}\right)\right) \text {. }
$$

Remark 1.4: Let $E=F$ and $X=Y$ in Definition 1.3. If $\phi: X \times X \rightarrow \mathbb{R}$ is convex (resp., concave) on $Y$, clearly $\phi$ is quasi-convex (resp., quasi-concave) on $Y$. When $\phi: X \times X \rightarrow \mathbb{R}$ is $\gamma$ diagonally quasi-convex (resp., quasi-concave) on $Y$ then $\phi$ is $\gamma$-generalized quasi-convex (resp., quasi-concave) on $Y$, where $\gamma:=\inf _{x \in X} \phi(x, x)$ (resp., $\gamma:=\sup _{x \in X} \phi(x, x)$.

The following result is a combination of Proposition 2 and Theorem 3.1 of Chang and Zhang [3] and it will be used in the study of Section 2 .

Proposition 1.5: Let $X$ and $Y$ be a non-empty convex subsets of topological vector spaces $E$ and $F$, respectively, and $\gamma \in \mathbb{R}$ a fixed constant. Suppose $\phi: X \times Y \rightarrow \mathbb{R}$ is a real-valued function. Then the set-valued mapping $G: Y \rightarrow \mathscr{P}(X)$, defined by

$$
G(y):=\{x \in X: \phi(x, y) \leq \gamma\}(\text { resp., } G(y):=\{x \in X: \phi(x, y) \geq \gamma\})
$$

for each $y \in Y$, is a generalized KKM mapping if and only if the function $\phi$ is $\gamma$-generalized quasi-concave (resp., quasi-convex) on $Y$. Moreover, if the mapping $G$ is finitely closed (i.e., for every finite-dimensional subspace $L$ of $F$, the set $G(x) \cap L$ is relatively closed in the relative Euclidean topology of $L$ for each $x \in X)$, then the family $\{G(x): x \in X\}$ has the finite intersection property if and only if the set-valued mapping $G$ defined above is a generalized KKM mapping.

\section{New Deterministic Variational Inequalities and Existence Theorems of Saddle Points in Topological Vector Spaces}

In this section, with the help of the concept of the generalized KKM mapping, we have established a general variational inequality with weaker convexity condition. This new variational inequality is then used to derive an existence theorem of saddle points for a realvalued function defined in topological vector spaces. Our the rems include a number of corresponding results in the literature as special cases (e.g., see [1], [3-4], [9], [12-13]).

Theorem 2.1: Let $X$ and $Y$ be non-empty convex subsets of topological vector spaces $E$ and $F$, respectively and $\gamma \in \mathbb{R}$ a fixed constant. Suppose two real-valued functions $\phi, \psi: X \times Y \rightarrow \mathbb{R}$ satisfy the following conditions:

(1) $\phi(x, y) \leq \psi(x, y)$ for each $(x, y) \in X \times Y$;

(2) for each fixed $x \in X$, the mapping $y \mapsto \phi(x, y)$ is lower semicontinuous on each non-empty compact subset $C$ of $Y$;

(3) there exist a non-empty compact subset $X_{0}$ of $X$, a non-empty compact convex subset $Y_{0}$ of $Y$ and a non-empty compact subset $K_{0}$ of $Y$ such that for each non-empty subset $\left(x_{1}, \ldots, x_{n}\right\} \subset X$, there exists a non-empty finite subset $\left\{y_{1}, \ldots, y_{n}\right\} \subset Y$ satisfying that the restriction of $\psi$ to $\operatorname{co}\left(X_{0} \cup\left\{x_{1}, \ldots, x_{n}\right\}\right) \times \operatorname{co}\left(Y_{0} \cup\left\{y_{1}, \ldots, y_{n}\right\}\right)$ is $\gamma$-generalized quasiconcave on $\operatorname{co}\left(X_{0} \cup\left\{x_{1}, \ldots, x_{n}\right\}\right)$;

(4) for each $y \in Y \backslash K$, there exists $x \in X_{0}$ such that $\phi(x, y)>\gamma$.

Then there exists $y \in K$ such that 


$$
\sup _{x \in X} \phi(x, y) \leq \gamma
$$

Proof: In order to reach the conclusion it suffices to show that the family $\{[y \in K$ : $\phi(x, y) \leq \gamma]: x \in X\}$ has the finite intersection property. By condition (3), for each non-empty finite subset $\left\{x_{1}, \ldots, x_{n}\right\}$ of $X$, there exists a non-empty finite subset $\left\{y_{1}, \ldots, y_{n}\right\}$ of $Y$ such that the mapping $\psi: D_{1} \times D_{2} \rightarrow \mathbb{R}$ is $\gamma$-generalized quasi-concave on $D_{1}$, where $D_{1}:=\operatorname{co}\left(X_{0} \cup\right.$ $\left\{x_{1}, \ldots, x_{n}\right\}$ and $D_{2}:=c o\left(Y_{0} \cup\left\{y_{1}, \ldots, y_{n}\right\}\right)$. Let us define two mappings $T_{1}, T_{2}: D_{1} \rightarrow \mathscr{P}\left(D_{2}\right)$ by

$$
T_{1}(x):=\left\{y \in D_{2}: \psi(x, y) \leq \gamma\right\}
$$

and

$$
T_{2}(x):=\left\{y \in D_{2}: \phi(x, y) \leq \gamma\right\}
$$

for each $x \in X$. Note that for each $y \in Y$, the mapping $x \rightarrow \psi(x, y)$ is $\gamma$-generalized from $D_{1} \times D_{2}$ to $\mathbb{R}$ so that $T_{1}(x)$ is non-empty for each $x \in D_{1}$. Moreover, $T_{1}$ is a generalized KKM mapping by Proposition 1.5. Therefore the family $\left\{T_{1}(x): x \in D_{1}\right\}$ has the finite intersection property by applying Proposition 1.5 again. As $T_{1}(x) \subset T_{2}(x)$ and $T_{2}(x)$ are non-empty compact subsets for each $x \in X$, it follows that $\bigcap_{x \in D_{1}} T_{2}(x) \neq \emptyset$. Taking any fixed $y \in \bigcap_{x \in D_{1}} T_{1}(x)$ we have that $y \in K$ by condition (4). Now define a mapping, $G: X \rightarrow \mathscr{P}(Y)$ by

$$
G(x):=\{y \in K: \phi(x, y) \leq \gamma\}
$$

for each $x \in X$. Then the family $\{G(x): x \in X\}$ has the finite intersection property. Note that $G(x)$ is compact so that $\bigcap_{x \in X} G(x) \neq \emptyset$. Taking any fixed $y^{*} \in \bigcap_{x \in X} G(x)$, we have $\sup _{x \in X} \phi\left(x, y^{*}\right) \leq \gamma$ and the conclusion follows.

We note that non-compact conditions (3) and (4) of Theorem 2.1 are different from the noncompact conditions which were posed by Chang and Zhang [3, Theorem 3.4]. In the case $E=F$ and $X=Y$ in Theorem 2.1, it still includes Theorem 3 of Shih and Tan [9], Theorem 6 of Fan [4], Theorem 2 of Allen [1], Theorem 1 of Yen [13], and Tarafdar [12] as special cases.

As an immediate consequence of Theorem 2.1, we have the following variational inequality which improves the well-known Ky Fan minimax inequality in several aspects (e.g., see Aubin [2]):

Corollary 2.2: Let $X$ be a non-empty convex subset of a topological vector space. Suppose that $f: X \times X \rightarrow \mathbb{R}$ is a real-valued function such that

(a) for each fixed $y \in X$, the mapping $x \rightarrow f(x, y)$ is lower semi-continuous on each nonempty compact subset $C$ of $X$;

(b) for each $A \in \mathcal{F}(X)$ and for each $x \in \operatorname{co}(A), \min _{y \in A} f(x, y) \leq 0$;

(c) there exists a non-empty compact subset $K$ of $X$ and a non-empty convex compact subset $X_{0}$ of $X$ such that for each $x \in X \backslash K$, there exists $y \in X_{0}$ with $f(x, y)>0$.

Then there exists $x \in X$ such that

$$
\sup _{y \in X} f(x, y) \leq 0
$$

Considering another application of Theorem 2.1, we obtain the following existence theorem of saddle points for a real-valued function defined on topological vector spaces.

Theorem 2.3: Let $X$ and $Y$ be non-empty convex subsets of topological vector spaces $E$ and $F$, respectively, and $\gamma \in \mathbb{R}$ a fixed constant. Suppose $\phi: X \times Y \rightarrow \mathbb{R}$ is a real-valued function satisfying 
(1) for each $x \in X$, the mapping $y \mapsto \phi(x, y)$ is lower semicontinuous on each non-empty compact subset $C$ of $Y$; and for each fixed $y \in Y$, the mapping $x \mapsto \phi(x, y)$ is upper semicontinuous on each non-empty compact subset $C$ of $X$;

(2) there exist non-empty compact convex subsets $X_{0}, X_{1}$ of $X$, non-empty compact convex subsets $Y_{0}, Y_{1}$ of $Y$, a non-empty compact (not necessarily convex) subset $K$ of $Y$ and a non-empty compact (not necessarily convex) subset $W$ of $X$ such that:

$(2)_{a}$ for each non-empty finite subset $\left\{x_{1}, \ldots, x_{n}\right\} \subset X$, there exists a non-empty finite subset $\left\{y_{1}, \ldots, y_{n}\right\}$ of $Y$ such that the restriction of $\phi$ to $\operatorname{co}\left(X_{0} \cup\left\{x_{1}, \ldots, x_{n}\right\}\right) \times \operatorname{co}\left(Y_{0} \cup\right.$ $\left.\left\{y_{1}, \ldots, y_{n}\right\}\right)$ is $\gamma$-generalized quasi-concave on $\operatorname{co}\left(x_{0} \cup\left\{x_{1}, \ldots, x_{n}\right\}\right)$; and

$(2)_{b}$ for each non-empty finite subset $\left\{y_{1}, \ldots, y_{n}\right\}$ in $Y$, there exists a non-empty finite subset $\left\{x_{1}, \ldots, x_{n}\right\}$ in $X$ with that the restriction of $\phi$ to $\operatorname{co}\left\{X_{1} \cup\left\{x_{1}, \ldots, x_{n}\right\}\right) \times \operatorname{co}\left(Y_{1} \cup\right.$ $\left.\left\{y_{1}, \ldots, y_{n}\right\}\right)$ is $\gamma$-generalized quasi-convex on $\operatorname{co}\left(Y_{1} \cup\left\{y_{1}, \ldots, y_{n}\right\}\right)$;

for each $y \in Y \backslash Y$, there exists $x \in X_{0}$ such that $\phi(x, y)>\gamma$ and for each $x \in X \backslash W$, there exists $y \in Y_{1}$ such that $\phi(x, y)<\gamma$.

Then $\phi$ has a saddle point $(\bar{x}, \bar{y}) \in X \times Y$; i.e., the following equality holds:

$$
\sup _{x \in X} \inf _{y \in Y} \phi(x, y)=\phi(\bar{x}, \bar{y})=\gamma=\underset{y \in Y}{\inf } \underset{x \in X}{\sup } \phi(x, y) .
$$

Proof: Let $\phi(x, y):=\psi(x, y)$ for each $(x, y) \in X \times Y$ in Theorem 2.1. Then Theorem 2.1 implies that there exists $\bar{y} \in Y$ such that

$$
\sup _{x \in X} \phi(x, \bar{y}) \leq \gamma
$$

Let $\phi_{1}(x, y)=-\psi(y, x)$ for each $(x, y) \in X \times Y$. Then $\phi_{1}$ satisfies all hypotheses of Theorem 2.1. Applying 2.1, there exists $\bar{x} \in X$ such that

$$
\sup _{y \in Y}-\psi(\bar{x}, y) \leq-\gamma
$$

Combining inequalities (1) and (2), we have

$$
\phi(x, \bar{y}) \leq \phi(\bar{x}, \bar{y}) \leq \phi(\bar{x}, y)
$$

for each $(x, y) \in X \times Y$. Thus,

$$
\inf _{y \in Y} \sup _{x \in C} \phi(x, y) \leq \sup _{x \in X} \phi(x, \bar{y}) \leq \gamma \leq \inf _{y \in Y} \phi(\bar{x}, y) \leq \sup _{x \in X} \inf _{y \in Y} \phi(x, y),
$$

which shows that $(\bar{x}, \bar{y})$ is a saddle point of $\phi$, i.e.,

$$
\inf _{y \in Y} \sup _{x \in X} \phi(x, y)=\phi(\bar{x}, \bar{y})=\gamma=\sup _{x \in X} \inf _{y \in Y} \phi(x, y)
$$

and we complete the proof.

Setting $E=F, X=Y, X_{0}=Y_{0}$, and $X_{1}=Y_{1}$ in Theorem 2.3, we have the following corollary which improves Theorem 5.1 of Chang and Zhang [3].

Corollary 2.4: Let $X$ be a non-empty convex subset of a topological vector space E. Suppose $\phi: X \times X \rightarrow \mathbb{R}$ is such that:

(a) for each fixed $x \in X$, the mapping $y \rightarrow \phi(x, y)$ is lower semi-continuous on each nonempty compact subset $C$ of $X$; and for each fixed $y \in X$, the mapping $x \rightarrow \phi(x, y)$ is upper semi-continuous on each non-empty compact subset $C$ of $X$;

(b) for each $A \in \mathscr{F}(X)$, each $x \in \operatorname{co}(A)$ and each $y \in \operatorname{co}(A), \quad \min _{y \in A} \phi(x, y) \leq 0$ and $\max _{x \in A} \phi(x, y)>0$; 
(c) there exist two non-empty convex compact subsets $X_{0}, X_{1}$ of $X$ and two non-empty compact (not necessarily convex) subsets $K_{0}, K_{1}$ of $X$ such that for each $x \in X \backslash K_{0}$, there exists $y \in X_{0}$ with $\phi(x, y)>0$; and for each $y \in X \backslash K_{1}$, there exists $x \in X_{1}$ such that $f(x, y)<0$.

Then $\phi$ has a saddle point $(\bar{x}, \bar{y}) \in X \times X$, i.e., $\phi(x, \bar{y}) \leq \phi(\bar{x}, \bar{y}) \leq \phi(\bar{x}, y)$ for each $(x, y) \in$ $X \times X$ and

$$
\sup _{x \in X} \inf _{y \in X} \phi(x, y)=\phi(\bar{x}, \bar{y})=0=\underset{y \in X}{\inf } \underset{x \in X}{\sup } \phi(x, y)
$$

\section{Random Variational Inequalities and Random Best Approximation Theorems}

By employing a measurable selection theorem of Himmelberg [5] and our variational inequality of Section 2, a random variational inequality is presented. As an application of our random variational inequality, we derive a random best approximation theorem which is a stochastic version of the best approximation theorem of Fan [4].

Let $X$ be a non-empty subset of a topological vector space $E$ and $f: \Omega \times X \times X \rightarrow \mathbb{R U}$ $\{-\infty,+\infty\}$ an extended real-valued function, where $(\Omega, \Sigma)$ is a measurable space. Then a single-valued measurable mapping $g: \Omega \rightarrow X$ is said to be a random variational solution for the function $f$ provided that

$$
\sup _{y \in X} f(\omega, g(\omega), y) \leq 0
$$

for all $\omega \in \Omega$. It is clear that if $f$ has a random variational solution $g$, the operator $f(\omega, \cdot, \cdot)$ has at least one variational solution as $\sup _{y \in X} f(\omega, g(\omega), y) \leq 0$ for each fixed $\omega \in \Omega$. However, the following simple example illustrates that the converse does not hold true in general, unless $f$ satisfies certain measurable conditions.

Example 3.1: Let $\Omega=X=[0,1], \Sigma$ the $\sigma$-algebra of Lebesgue measurable subsets of $[0,1]$, and $A$ a non-Lebesgue measurable subset of [0,1]. Define $f: \Omega \times X \times X \rightarrow \mathbb{R} \cup\{-\infty,+\infty\}$ by

$$
f(\omega, x, y)=\left\{\begin{array}{cc}
(x-1) \cdot y, & \text { if }(\omega, x, y) \in A \times X \times X ; \\
x \cdot y, & \text { otherwise. }
\end{array}\right.
$$

Then for each fixed $\omega \in \Omega, f(\omega, \cdot, \cdot)$ has a unique variational solution $\psi$, which is

$$
\psi(\omega)= \begin{cases}\{1\}, & \text { if } \omega \in A \\ \{0\} ; & \text { otherwise }\end{cases}
$$

However, $f$ does not have any random variational solution as $\psi$ is not measurable.

In what follows, we shall present one existence theorem of random variational solutions when $f$ satisfies certain continuous and measurable conditions. We recall a measurable selection theorem of Himmelberg [5, Theorem 5.6] which is stated as follows:

Theorem 3.A: Let $(\Omega, \Sigma)$ be a measurable space and $X$ a separable metric space. Suppose $F: \Omega \rightarrow \mathscr{P}(X)$ is a mapping with complete values. Then $F$ is weakly measurable if and only if there exists a countable family $\left\{g_{i}\right\}_{i=1}^{\infty}$ of measurable selection for $F$ such that $F(\omega)=\overline{\left\{g_{i}(\omega): 1,2, \ldots\right\}}$ for all $\omega \in \Omega$. If $X$ is also $\sigma$-compact, $F$ only needs to have closed values.

Then we obtain the following existence theorem for random variational solutions: 
Theorem 3.1: Let $(\Omega, \Sigma)$ be a measurable space and $X$ a non-empty separable metrizable convex subset of a Hausdorff topological vector space. Suppose $f: \Omega \times X \times X \rightarrow \mathbb{R} \cup\{-\infty,+\infty\}$ such that:

(a) $\quad \omega \mapsto f(\omega, x, y)$ is measurable for each fixed $(x, y) \in X \times X$;

(b) $x \mapsto f(\omega, x, y)$ is compactly continuous for each fixed $(\omega, y) \in \Omega \times X$ (i.e., $x \mapsto f(\omega, x, y)$ is continuous on each non-empty compact subset of $X)$;

(c) $\quad y \mapsto f(\omega, x, y)$ is lower semicontinuous for each fixed $(\omega, x) \in \Omega \times X$;

(d) for each $A \in \mathcal{F}(X)$ and each $x \in \operatorname{co}(A), \min _{y \in A} f(\omega, x, y) \leq 0$ for all $\omega \in \Omega$; and

(e) there exists a non-empty compact subset $K$ of $X$ and a non-empty compact and convex subset $X_{0}$ of $X$ such that for any $x \in X \backslash K$ there exists $y \in K_{0}$ satisfying $f(\omega, x, y)>0$ for all $\omega \in \Omega$.

Then there exists a countable measurable family $\left\{g_{i}\right\}_{i=1}^{\infty}$ from $\Omega$ to $K$ such that

$$
\sup _{y \in X} f\left(\omega, g_{i}(\omega), y\right) \leq 0
$$

for each $g_{i}$ and all $\omega \in \Omega$.

Proof: Define a set-valued mapping $\psi: \Omega \rightarrow \mathscr{P}(K)$ by

$$
\psi(\omega):=\left\{x \in K: \sup _{y \in X} f(\omega, x, y) \leq 0\right\}
$$

for each $\omega \in \Omega$. Then $\psi(\omega)$ is a non-empty closed subset of $K$ for each $\omega \in \Omega$ by Corollary 2.2. We claim that $\psi: \Omega \rightarrow \mathscr{P}(K)$ is measurable. Let $D:=\left\{x_{n}: n=1, \ldots\right\}$ be a countable dense subset of $K$, since $K$ is metrizable and compact. For each $n \in \mathbb{N}$, define $\psi_{n}: \Omega \rightarrow \mathscr{P}(K)$ by

$$
\psi_{n}(\omega):=\left\{x \in K: f\left(\omega, x, x_{n}\right) \leq 0\right\}
$$

for each $\omega \in \Omega$. Due to the lower semicontinuity of $y \mapsto f(\omega, x, y), \psi(\omega)=\bigcap_{n=1}^{\infty} \psi_{n}(\omega)$ for each $\omega \in \Omega$. Note that $\psi_{n}$ has non-empty compact values for each $n \in \mathbb{N}$. In order to prove $\psi$ to be measurable, it suffices to show that $\psi_{n}$ is measurable (by Theorem 4.1 of Himmelberg [5]). Let $C$ be any non-empty closed subset of $K$ and $C_{0}$ be its countable dense subset. From condition $(b)$, $x \mapsto f(\omega, x, y)$ is continuous on $K$ and we have

$$
\begin{gathered}
\psi_{n}^{-1}(C)=\left\{\omega \in \Omega: \psi_{n}(\omega) \cap C \neq \emptyset\right\} \\
=\bigcup_{x \in C}\left\{\omega \in \Omega: f\left(\omega, x, x_{n}\right) \leq 0\right\} \\
=\bigcap_{m=1}^{\infty}\left\{\bigcup_{x_{i} \in C_{0}}\left[\omega \in \Omega: f\left(\omega, x_{i}, x_{n}\right)<\frac{1}{m}\right]\right\}
\end{gathered}
$$

which is measurable by condition $(a)$. Indeed, if $\omega \in \psi_{n}^{-1}(C)$, there exists $x \in C$ such that $f\left(\omega, x, x_{n}\right) \leq 0<\frac{1}{m}$ for all $m \in \mathbb{N}$. Since $x \rightarrow f\left(\omega, x, x_{n}\right)$ is continuous, there exists $x_{m} \in C_{0}$ such that $f\left(\omega, x_{m}, x_{n}\right)<\frac{1}{m}$. Hence,

$$
\psi_{n}^{-1}(C) \subseteq \bigcap_{m=1}^{\infty}\left\{\bigcup_{x_{i} \in C_{0}}\left[\omega: f\left(\omega, x_{i}, x_{n}\right)<\frac{1}{m}\right]\right\} .
$$

Now suppose $\omega \in \bigcap_{m=1}^{\infty}\left\{\bigcup_{x_{i} \in C_{0}}\left[\omega: f\left(\omega, x_{i}, x_{n}\right)<\frac{1}{m}\right]\right\}$. For each $m \in \mathbb{N}$, there exists $x_{m} \in C_{0}$ such that $f\left(\omega, x_{m}, x_{n}\right)<\frac{1}{m}$. As $C_{0} \subset C$ and $C$ is compact, without loss of generality, we assume that $\left\{x_{m}\right\}_{m \in \mathbb{N}}$ converges to $x_{0} \in C$. The lower semicontinuity of $x \mapsto f\left(\omega, x, x_{n}\right)$ implies that

Thus,

$$
f\left(\omega, x_{0}, x_{n}\right) \leq \liminf _{m \rightarrow \infty} f\left(\omega, x_{m}, x_{n}\right) \leq 0 .
$$

$$
\psi_{n}^{-1}(C)=\bigcap_{m=1}^{\infty}\left\{\bigcup_{x_{i} \in C_{0}}\left[\omega \in \Omega: f\left(\omega, x_{i}, x_{n}\right)<\frac{1}{m}\right]\right\}
$$


which shows that $\psi_{n}$ is measurable, and so is the mapping $\psi$ by Theorem 4.1 of Himmelberg [5]. By Theorem 3.A, there exists a countable family of measurable selections $\left\{g_{i}\right\}_{i=1}^{\infty}$ of $\psi$ from $\Omega$ to $K$ such that $\psi(\omega)=\overline{\left\{g_{i}(\omega): i=1,2, \ldots\right\}}$. From the definition of $\psi$, it follows that

$$
\sup _{y \in C} f\left(\omega, g_{i}(\omega), y\right) \leq 0
$$

for each $g_{i}$ and all $\omega \in \Omega$. Thus, the proof is complete.

Let $A$ and $B$ be two non-empty subsets of a normed space $(E,\|\cdot\|)$. We denote by $d(A, B):=\inf \{\|x-y\|: x \in A$ and $y \in B\}$ the distance between $A$ and $B$.

As an application of Theorem 3.1, we have the following random best approximation theorem which is a stochastic version of Fan's best approximation theorem [4, Theorem 2].

Theorem 3.2: Let $(\Omega, \Sigma)$ be a measurable space and $X$ a non-empty separable convex subset of a normed space $(E,\|\cdot\|)$. Suppose $\psi: \Omega \times X \rightarrow \mathscr{P}(E)$ is a randomly continuous mapping with non-empty compact and convex values. Moreover, assume that there exists a non-empty convex compact subset $X_{0}$ of $X$ and a non-empty compact subset $K$ of $X$ such that for each $x \in X \backslash K$, there exists $y \in X_{0}$ with inf $u \in \psi(\omega, x)\|x-u\|<i n f_{u \in \psi(\omega, x)}\|x-u\|$ for all $\omega \in \Omega$. Then there exists a countable measurable family $\left\{g_{i}\right\}_{i=1}^{\infty}$ from $\Omega$ to $K$ such that

$$
\inf _{u \in \psi\left(\omega, g_{i}(\omega)\right)}\left\|g_{i}(\omega)-u\right\|=d\left(X, \psi\left(\omega, g_{i}(\omega)\right)\right)
$$

for each $g_{i}$ and all $\omega \in \Omega$.

Proof: In order to apply Theorem 3.1 , we define $f: \Omega \times X \times X \rightarrow \mathbb{R} \cup\{-\infty,+\infty\}$ by

$$
f(\omega, x, y)=\inf _{z \in \psi(\omega, x)}\|z-x\|-\inf _{z \in \psi(\omega, x)}\|z-y\|
$$

for each $(\omega, x, y) \in \Omega \times X \times X$. Because $\psi(\omega, x)$ is non-empty compact, the mapping $(\omega, x, y) \mapsto$ $f(\omega, x y)$ is randomly continuous by Lemma 3 of Sehgal and Singh [10]. Now we show that the function $f$ satisfies all of Theorem 3.1. Fixing each $\omega \in \Omega$, for $A \in \mathscr{F}(X)$ and each $x \in \operatorname{co}(A)$, it must hold that $\min _{y \in A} f(\omega, x, y) \leq 0$; otherwise there exist $A:=\left\{y_{i}, \ldots, y_{n}\right\} \in \mathscr{F}(X)$ and $x=$ $\sum_{i=1}^{n} \lambda_{i} y_{i} \in \operatorname{co}(A)$, where $\lambda_{i}, \ldots, \lambda_{n} \geq 0$ with $\sum_{i=1}^{n} \lambda_{i}=1$ such that $f\left(\omega, x, y_{i}\right)>0$ for all $i=1, \ldots, n$. Since $F(\omega, x)$ is compact, there exists $z_{i} \in \psi(\omega, x)$ such that $\left\|z_{i}-y_{i}\right\|=$ $\inf _{z \in \psi(\omega, x)}\left\|z-y_{i}\right\|$ for $i=1,2, \ldots, n$, i.e.,

$$
f\left(\omega, x, y_{i}\right)=\inf _{z \in \psi(\omega, x)}\|z-x\|-\inf _{z \in \psi(\omega, x)}\left\|z-y_{i}\right\|=\inf _{z \in \psi(\omega, x)}\|z-x\|-\left\|z_{i}-y_{i}\right\|
$$

for each $i=1, \ldots, n$. Let $z_{0}=\sum_{i=1}^{n} \lambda_{i} z_{i}$. Then $z_{0} \in \psi(\omega, x)$ as $F(\omega, x)$ is convex. It follows that

$$
\begin{gathered}
0<f\left(\omega, x, y_{i}\right)=\inf _{z \in \psi(\omega, x)}\|z-x\|-\inf _{z \in \psi(\omega, x)}\left\|z-y_{i}\right\| \\
\leq\left\|z_{0}-x\right\|-\inf _{z \in \psi(\omega, x)}\left\|z-y_{i}\right\| \leq \sum_{i=1}^{n} \lambda_{i}\left\|z_{i}-y_{i}\right\|-\inf _{z \in \psi(\omega, x)}\left\|z-y_{i}\right\| \\
=0,
\end{gathered}
$$

which is not true. Thus, $f$ satisfies all the conditions of Theorem 3.1. By Theorem 3.1, there exists countable measurable mappings $\left\{g_{i}\right\}_{i \in \mathbb{N}}$ from $\Omega$ to $K$ such that

$$
\sup _{y \in X} f\left(\omega, g_{i}(\omega), y\right) \leq 0
$$


for each $g_{i}$ and all $\omega \in \Omega$, and so that

$$
d\left(\psi\left(\omega, g_{i}(\omega)\right)=d\left(\psi\left(\omega, g_{i}(\omega)\right), X\right)\right.
$$

for all $\omega \in \Omega$.

Theorem 3.2 includes Theorem 2 of Sehgal and Singh [10] as a special case.

We would like to observe that some other kinds of random best approximation theorems have been established (e.g., see Tan and Yuan [11], Yuan [14] and the references contained therein) when the measurable space $(\Omega, \Sigma)$ has the property that $\Sigma$ is a Suslin family. Note that not all $\sigma$ algebra $\Sigma$ s are Suslin families (the definition of Suslin family can be found in either [11] or [14]. For example, the $\sigma$-algebra which consists of all Lebesgue measurable subsets of $[0,1]$ is not one (e.g., see Royden [8]). Thus, Theorem 3.2 is independent of those random best approximation theorems in the literature, such as [11] and [14].

\section{Random Fixed Point Theorems}

As applications of the random best approximation Theorem 3.2, we prove some random fixed point theorems.

Theorem 4.1: Let $(\Omega, \Sigma)$ be a measurable space and $X$ a non-empty complete separable convex subset of a normed space $(E \cdot)$. Suppose $\psi: \Omega \times X \rightarrow \mathscr{P}(E)$ is a randomly continuous mapping with non-empty compact and convex values such that:

(a) there exist a non-empty convex compact subset $X_{0}$ of $X$ and a non-empty compact subset $K$ of $X$ such that for each $y \in X \backslash K$ there exists $x \in X_{0}$ with inf $_{u \in \psi(\omega, y)}\|x-u\|<$ inf $_{u \in \psi(\omega, y)}\|y-u\|$ for each $\omega \in \Omega$; and $\psi$ satisfies one of the following conditions:

(i) for each fixed $\omega \in \Omega$, each $x \in K$ with $x \neq \psi(\omega, x)$, there exists $y \in I_{X}(x):=$ $\{x+c(z-x)$ for some $z \in X$ and some $c>0\}$ such that inf $u \in \psi(\omega, x)\|y-u\|<$ inf $_{u \in \psi(\omega, x)}\|x-u\|$; or

(ii) $\quad \psi$ is weakly inward (i.e., for each $\omega \in \Omega, \psi(\omega, x) \cap \overline{I_{X}(x)} \neq \emptyset$ for each $x \in K$ ).

Then $\psi$ has a random fixed point.

Proof: By Theorem 3.2, there exists a countable measurable family $\left[g_{i}\right\}_{i=1}^{\infty}$ from $\Omega$ to $K$ such that

$$
\inf _{u \in \psi\left(\omega, g_{i}(\omega)\right)}\left\|g_{i}(\omega)-u\right\|=d\left(\psi\left(\omega, g_{i}(\omega)\right), X\right)
$$

for each $g_{i}$ and all $\omega \in \Omega$. We now prove that each $g_{i}$ is a random fixed point of $\psi$.

Suppose $\psi$ satisfies $(b)(i)$. If there exists some $\omega \in \Omega$ such that $g_{i}(\omega) \notin \psi\left(\omega, g_{i}(\omega)\right)$, by our assumption $(b)(i)$, there exists $y \in I_{X}\left(g_{i}(\omega)\right)$ such that

$$
\inf _{u \in \psi\left(\omega, g_{i}(\omega)\right)}\|y-u\|<\inf _{u \in \psi\left(\omega, g_{i}(\omega)\right)}\left\|g_{i}(\omega)-u\right\|
$$

Note that $y \in I_{X}\left(g_{i}(\omega)\right)$, there exists $z \in X$ and $c>0$ such that $y=g_{i}(\omega)+c\left(z-g_{i}(\omega)\right)$, so that $y \notin X$; otherwise a contradiction to the choice of $g_{i}(\omega)$ would result. Without loss of generality, we assume that $c>1$. Then $z:=y / c+(1-1 / c) g_{i}(\omega)=(1-\beta) y+\beta g_{i}(\omega)$, where $\beta=1-1 / c$ and $0<\beta<1$. Let $\omega \in \psi\left(\omega, g_{i}(\omega)\right)$ such that $\left\|g_{i}(\omega)-\omega\right\|=\inf _{u \in \psi\left(\omega, g_{i}(\omega)\right)}\left\|g_{i}(\omega)-u\right\|=$ $d\left(\psi\left(\omega, g_{i}(\omega)\right), X\right)$. Then, 


$$
\begin{gathered}
\|z-w\| \leq(1-\beta)\|y-w\|+\beta\left\|g_{i}(\omega)-w\right\| \\
<(1-\beta)\left\|g_{i}(\omega)-w\right\|+\beta\left\|g_{i}(\omega)-w\right\| \\
=\left\|g_{i}(\omega)-w\right\|=\inf _{u \in \psi\left(\omega, g_{i}(\omega)\right)}\left\|g_{i}(\omega)-u\right\| \\
=d\left(\psi\left(\omega, g_{i}(\omega)\right), X\right),
\end{gathered}
$$

and this contradicts the choice of $g_{i}(\omega)$. Therefore, $g_{i}(\omega) \in \psi\left(\omega, g_{i}(\omega)\right)$ for each $\omega \in \Omega$, i.e., $g_{i}$ is a random fixed point of $\psi$.

Let $\psi$ satisfy $(b)(i i)$ then, for each $\omega \in \Omega$ and each $x \in K$ with $x \neq \psi(\omega, x)$ there must exist $y \in I_{X}(x)$ such that $\inf _{u \in \psi(\omega, x)}\left\|y-u \mid<\inf _{u \in \psi(\omega, x)}\right\| x=u \|$ as $\psi(\omega, x) \cap \overline{I_{X}(x)} \neq \emptyset$ and $\psi$ is randomly continuous. Thus, $\psi$ satisfies the assumption $(i)$. Therefore, each $g_{i}$ is a random fixed point of $\psi$.

As an application of Theorem 4.1, we have the following random fixed point theorem.

Theorem 4.2: Let $(\Omega, \Sigma)$ be a measurable space and $X$ a non-empty complete separable convex subset of a normed space $(E,\|\cdot\|)$. Suppose $\psi: \Omega \times X \rightarrow \mathscr{P}(R)$ is a random continuous mapping with non-empty compact and convex values and there exist a non-empty compact convex subset $X_{0}$ of $X$ and a non-empty compact subset $K$ of $X$ such that

(a) for each $y \in X \backslash K$, there exists $x \in X_{0}$ such that inf $u \in \psi(\omega, y)\|x-u\|<$ inf $_{u \in \psi(\omega, y)}$ $\|y-u\|$ for all $\omega \in \Omega$;

(b) $\quad \psi$ satisfies $\psi(\omega, \partial K) \cap \bar{X} \neq \emptyset$ for all $\omega \in \Omega$.

Then $\psi$ has a random fixed point.

Proof: Since $\psi(\omega, \partial K) \cap \bar{X} \neq \emptyset$ for all $\omega \in \Omega, \psi$ satisfies condition $(b)(i i)$ of Theorem 4.1 due to the fact that $(i) \quad K \subset X \subset I_{X}(x), I_{K}(x) \subset I_{X}(x)$ and $(i i) \quad I_{K}(x)=E$ for each $x \in \operatorname{int} K$. Therefore, for each $x \in K, \psi(\omega, x) \cap I_{X}(x) \neq \emptyset$ for all $\omega \in \Omega$, and the conclusion follows from Theorem 4.1.

Remark 4.3: Theorem 4.2 improves the corresponding result of Sehgal and Singh [10].

\section{Acknowledgement}

The authors would like to thank Professor J. Dshalalow and an anonymous referee for carefully reading this manuscript and for helpful suggestions offered to lead the present version of this paper.

\section{References}

[1] Allen, G., Variational inequalities, complementarities problems, and duality theorems, $J$. Math. Anal. Appl. 58 (1977), 1-10.

[2] Aubin, J.-P., Mathematical Methods of Games and Economic Theory, (Revised Edition), North-Holland, Amsterdam, New York, Oxford 1982.

[3] Chang, S.S. and Zhang, Y., Generalized KKM theorem and variational inequalities, J. Math. Anal. Appl. 159 (1991), 108-223.

[4] Fan, K., Extensions of two fixed point theorems of F.E. Browder, Math. Z. 112 (1969), 234-240.

[5] Himmelberg, C.J., Measurable relations, Fund. Math. 87 (1975), 53-72. 
[6] Horvath, C., Contractibility and generalized convexity, J. Math. Anal. Appl. 156 (1991), 341-357.

[7] Lin, B.L. and Simons, S., editors, Nonlinear and convex analysis: Proceedings in Honor of Ky Fan, Marcel Dekker, Inc. 1987.

[8] Royden, H.L., Real Analysis, (2nd Edition), Macmillan, New York 1968.

[9] Shih, M.H. and Tan, K.K., A geometric property of convex sets with applications to minimax type inequalities and fixed point theorems, J. Austr. Math. Soc. A45 (1988), 169183.

[10] Sehgal, V.M. and Singh, S.P., On random approximations and a random fixed point theorem for set-valued mappings, Proc. Amer. Math. Soc. 95 (1985), 91-94.

[11] Tan, K.K. and Yuan, X.Z., On deterministic and random fixed points, Proc. Amer. Math. Soc. 119 (1992), 849-856.

[12] Tarafdar, E., On nonlinear variational inequalities, Proc. Amer. Math. Soc. 67 (1977), 9598.

[13] Yen, C.L., A minimax inequality and its applications to variational inequalities, Pacific J. Math. 97 (1981), 477-481.

[14] Yuan, X.Z., Non-compact random generalized games and random quasi-variational inequalities, J. Appl. Math. Stoch. Anal. 7 (1994), 467-486.

[15] Zhou, J.X. and Chen, G.N., Diagonal convexity conditions for problems in convex analysis and quasi-variational inequalities, J. Math. Anal. Appl. 132 (1988), 213-225. 


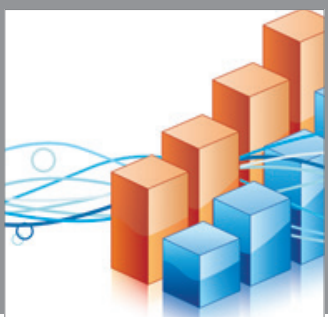

Advances in

Operations Research

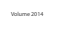

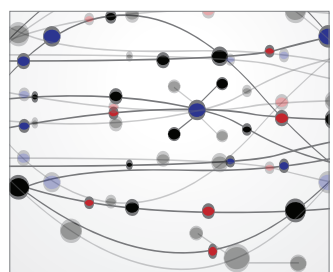

\section{The Scientific} World Journal
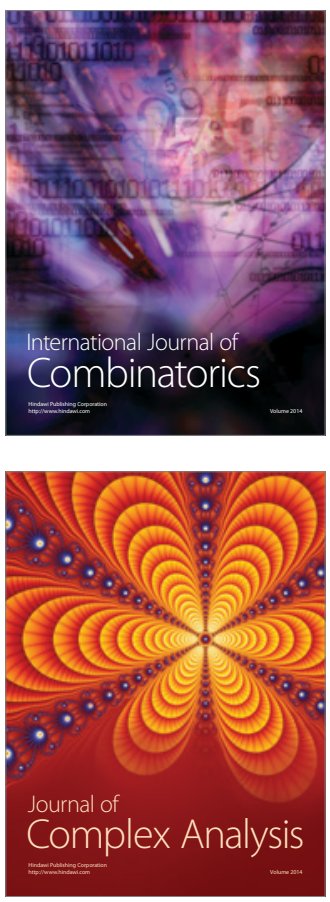

International Journal of

Mathematics and

Mathematical

Sciences
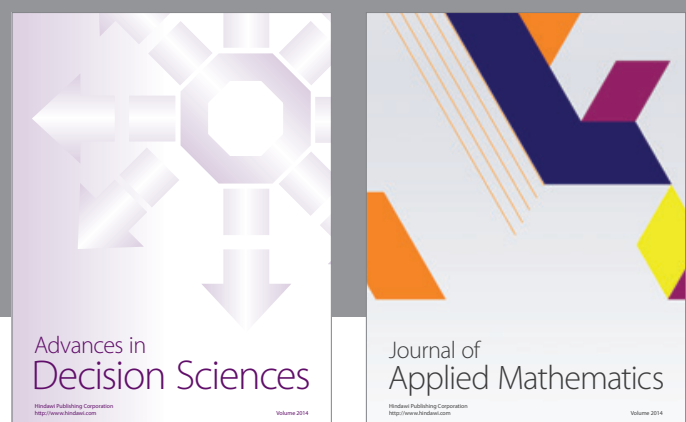

Journal of

Applied Mathematics
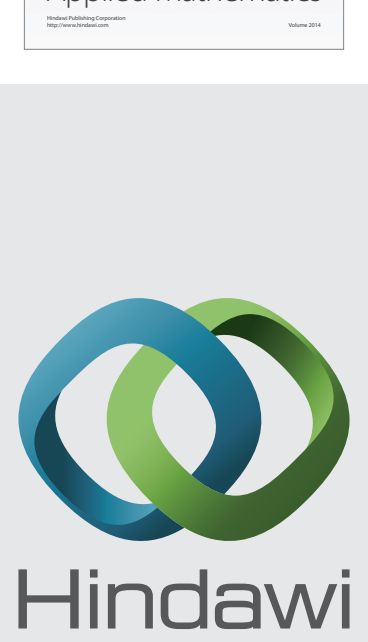

Submit your manuscripts at http://www.hindawi.com
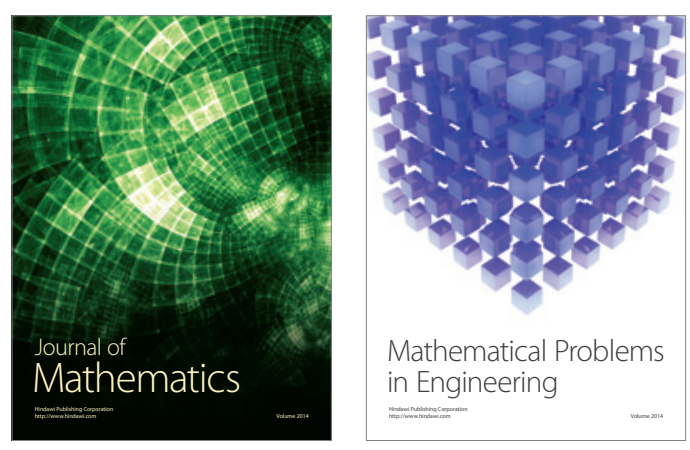

Mathematical Problems in Engineering
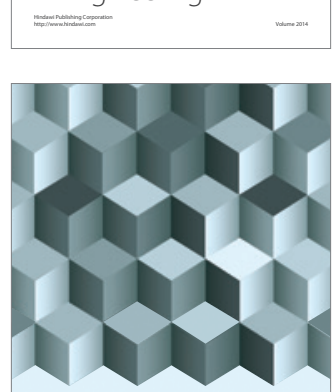

Journal of

Function Spaces
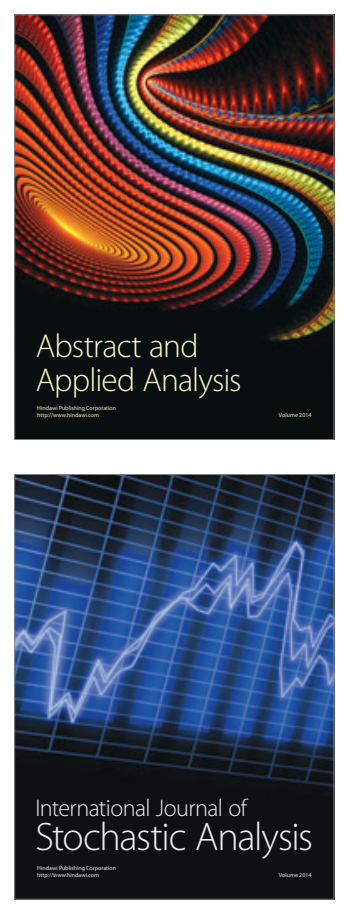

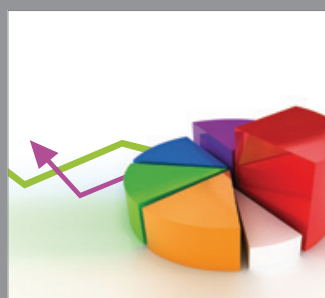

ournal of

Probability and Statistics

Promensencen
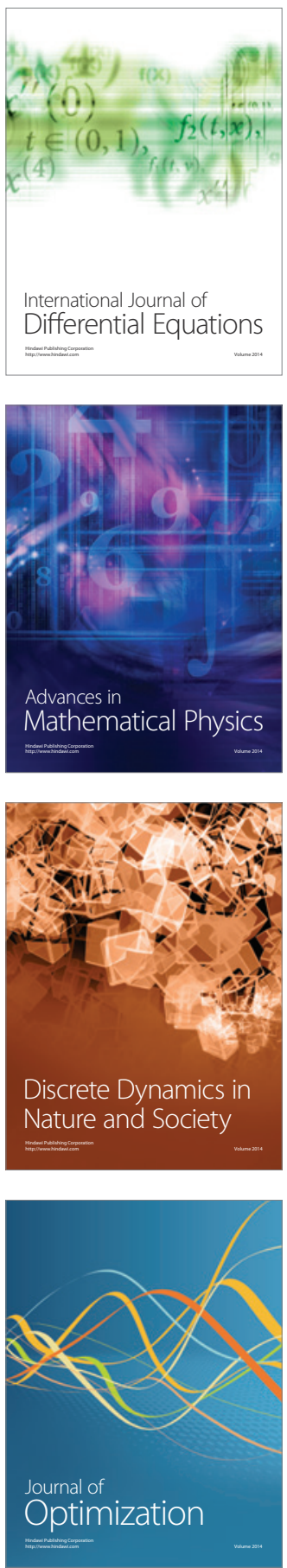